\title{
AMERICAN GINSENG IN REDUCING FATIGUE IN PATIENTS TREATED FOR HEAD AND NECK CANCER: A RANDOMIZED, DOUBLE-BLIND, PLACEBO CONTROLLED, MONO-CENTRE PHASE II STUDY
}

P. Di Pede ${ }^{1}$, R. Granata ${ }^{2}$, M. Guglielmo ${ }^{1}$, M. Ferrara ${ }^{1}$, C. Ripamonti ${ }^{1}, \underline{\text { P. Bossi }}{ }^{2}$

${ }^{1}$ Fondazione IRCCS Istituto Nazionale dei Tumori, Supportive Care in Cancer- Department OncologyEmatology, Milano, Italy

${ }^{2}$ Fondazione IRCCS Istituto Nazionale dei Tumori, Head and Neck Medical Oncology Department, Milano, Italy

\section{Introduction}

There are few data about fatigue treatment in Head and Neck cancer (HNC) patients (pts) during follow up (FU)

\section{Objectives}

To evaluate the efficacy of American Ginseng on fatigue reduction in HNC patients in FU

\section{Methods}

Consecutive eligible pts coming for planned follow-up visits were randomized $1: 1$ to receive American ginseng $1000 \mathrm{mg} /$ day or placebo for 8 weeks. Inclusion criteria were: curative intent treatment for primary HNC ended since more than 1 year, no evidence of disease; Brief Fatigue Inventory (BFI) score $\geq 4$, lack of overt clinical evidence of cognitive failure, no diagnosis of psychiatric disorder. Reduction in BFI score at the end of treatment was the primary endpoint. We conducted analysis according to intention to treat (ITT) and per protocol (PP)

\section{Results}

We enrolled 32 patients with the following characteristics: 22 males and 10 females, median age 55 years, mainly oropharyngeal cancer pts (15), of whom 12 with HPV positive cancer; median time since treatment end was 2.4 years. No significant differences in tumor site, HPV and gender were reported between treatment and placebo pts.

No major side effect was reported, except by 2 pts in treatment arm who interrupted treatment due to gastric intolerance. Mean difference in BFI score (after and before treatment) was 1.8 in ginseng group and 2.1 in placebo group $(p=n s)$, according to PP analysis, not different from ITT results ( $p=n s)$

\section{Conclusions}

Ginseng was quite tolerated, but not active in reducing fatigue in FU patients after treatment for HNC 